\title{
Scale-free primordial cosmology
}

\section{Citation}

Ijjas, Anna, Paul J. Steinhardt, and Abraham Loeb. 2014. "Scale-Free Primordial Cosmology." Physical Review D 89 (2). https://doi.org/10.1103/physrevd.89.023525.

\section{Permanent link}

http://nrs.harvard.edu/urn-3:HUL.InstRepos:41461270

\section{Terms of Use}

This article was downloaded from Harvard University's DASH repository, and is made available under the terms and conditions applicable to Other Posted Material, as set forth at http:// nrs.harvard.edu/urn-3:HUL.InstRepos:dash.current.terms-of-use\#LAA

\section{Share Your Story}

The Harvard community has made this article openly available.

Please share how this access benefits you. Submit a story.

Accessibility 


\title{
Scale-free primordial cosmology
}

\author{
Anna Ijjas \\ Max-Planck-Institute for Gravitational Physics (Albert- Einstein-Institute), 14476 Potsdam, \\ Germany and Rutgers University, New Brunswick, New Jersey 08901, USA \\ Paul J. Steinhardt ${ }^{\dagger}$ \\ Department of Physics and Princeton Center for Theoretical Science, Princeton University, \\ Princeton, New Jersey 08544, USA \\ Abraham Loeb \\ Harvard-Smithsonian Center for Astrophysics, Cambridge, Massachusetts 02138, USA
}

(Received 8 October 2013; published 28 January 2014)

\begin{abstract}
The large-scale structure of the Universe suggests that the physics underlying its early evolution is scalefree. This was the historic motivation for the Harrison-Zel'dovich-Peebles spectrum and for inflation. Based on a hydrodynamical approach, we identify scale-free forms for the background equation of state for both inflationary and cyclic scenarios and use these forms to derive predictions for the spectral tilt and tensor-to-scalar ratio of primordial density perturbations. For the case of inflation, we find three classes of scale-free models with distinct predictions. Including all classes, we show that scale-free inflation predicts tensor-to-scalar ratio $r>10^{-4}$. We show that the observationally favored class is theoretically disfavored because it suffers from an initial conditions problem and the hydrodynamical form of an unlikeliness problem similar to that identified recently for certain inflaton potentials. We contrast these results with those for scale-free cyclic models.
\end{abstract}

DOI: 10.1103/PhysRevD.89.023525

PACS numbers: $98.80 .-\mathrm{k}$

\section{INTRODUCTION}

The recent Planck satellite measurements [1-3], together with earlier observations from the Wilkinson Microwave Anisotropy Probe, Atacama Cosmology Telescope, South Pole Telescope, and other experiments [4], showed with high precision that the spectrum of primordial density fluctuations is nearly scale invariant, Gaussian, and adiabatic. These results suggest that the Universe is simple and the physics governing its early evolution on large scales is "scale-free." That is, the physics during that smoothing period in which the large-scale structure of the Universe is determined is governed by dynamical equations that entail no dimensionful macroscopic scales and yield power-law solutions.

Scale-freeness was first conjectured as a guiding cosmological principle over four decades ago and was the historic motivation for both the Harrison-Zel'dovichPeebles spectrum [5-7] and inflation [8-10]. In the intervening years, the principle seemed to lose favor as many baroque versions of inflationary (and other) models were proposed that explicitly introduce distinctive, scalesensitive features on large scales. The problem is that, without a guiding principle such as scale-freeness, literally any result for the spectral tilt, tensor-to-scalar ratio or other cosmological observables is possible. Some have

\footnotetext{
*aijjas@princeton.edu

steinh@princeton.edu

*aloeb@cfa.harvard.edu
}

emphasized this as an "attractive" feature of inflation on the grounds that the theory cannot be disproven (see for example [11]); but the other side of the coin is that this means the theory is entirely unpredictive.

Now that scale-freeness has substantial observational support, it is timely to examine how this guiding principle dramatically collapses the range of outcomes and makes cosmological theories like inflation meaningfully predictive. We use a hydrodynamical approach that is model independent, i.e., with no reference to scalar fields or potentials, to consider two well-known cosmological scenarios, the inflationary and cyclic (or ekpyrotic) theories of the Universe. We identify forms for the background equation of state during the cosmological smoothing phase in each case consistent with strict scale-freeness. We also consider variations that "weakly" break scale-freeness. We then derive generic predictions for the spectral tilt and tensor-to-scalar ratio of primordial density perturbations resulting from the scale-free principle.

A hydrodynamical approach has been applied earlier to inflationary and cyclic theories $[12,13]$, without explicitly assuming scale-freeness. The hydrodynamical approach is attractive since it is powerful and simple at the same time; it enables us to derive generic results (given the assumptions) and leads us to an intuitive understanding of the underlying physical phenomena. It is also closer to observation, in the sense that it is easier to determine the equation of state from astrophysical data than to determine the microphysics (scalar fields and potentials) that caused it. 
The goal of this paper is to show how the combination of the hydrodynamical approach and the principle of scalefreeness impose restrictions on cosmological scenarios and their predictions. For inflation, the combination reveals the existence of three distinct classes of scale-free scenarios. We show that the class favored by current experiment suffers from an initial conditions problem and a series of other problems, including a hydrodynamic equivalent of the unlikeliness problem identified recently for certain inflaton potentials [14]. For the cyclic scenarios, where smoothing occurs during a period of ultraslow (ekpyrotic) contraction, we find that there is only one class of scenarios and that none of the problems arise. In this analysis, we only consider a single contraction period without regard to whether the evolution repeats cyclically, so the same conclusions apply to bouncing cosmologies using ekpyrotic smoothing that have a single bounce or other variations.

For the cyclic (or other ekpyrotic) theories, most current versions use the entropic mechanism to generate curvature perturbations [15], which imposes the conceptual restriction that there be a two-component fluid to generate the perturbations. We find that handling two components rather than one in our approach is not a problem. We show that scale-freeness constrains the equations of state of both components, enabling us to derive generic predictions for the spectral tilt and tensor-to-scalar ratio analogous to the case of inflation.

We believe the approach adopted here based on scalefreeness and hydrodynamics provides what is arguably the predictions of the simplest, best-motivated, and observationally best-supported models of each given cosmological theory and sets a standard that can be applied to any scenario in which a smooth, i.e., scale-free background and nearly scale-invariant, adiabatic, and Gaussian perturbations are created at the same cosmological stage.

The paper is organized as follows. We begin in Sec. II by briefly reviewing the inflationary and cyclic (or ekpyrotic) scenarios and how they can create a scale-free background. To describe the background dynamics, in Sec. III we identify forms of the equation of state consistent with the principle of scale-freeness for the inflationary scenario. We demonstrate the existence of three distinct classes of scale-free solutions. Then, we use our background solutions to derive predictions for the spectral tilt and tensor-toscalar ratio of primordial density perturbations. We also consider cases with deviations from scale-freeness on unobservably small scales. Here and throughout the paper, our main aim is to make most generic statements from a minimal set of assumptions. In Sec. IV, we repeat the same type of analysis for the cyclic (ekpyrotic) model. We conclude in Sec. V by summarizing the constraints imposed by scale-freeness for both the inflationary and cyclic theories and comparing with constraints imposed by recent data.

\section{SCALE-FREENESS}

Both inflation and the cyclic (or ekpyrotic) theory were introduced to explain how inhomogeneous and anisotropic initial conditions can be made smooth and (spatially) flat, resulting in a scale-free universe. Inflation [8-10] accomplishes the feat with a phase of accelerated expansion occurring very shortly after the big bang. Alternatively, flatness and homogeneity can be achieved by an ekpyrotic smoothing phase [16,17], a period of ultraslow contraction before the big bang.

In both phases, the dynamics can be easily understood, using a hydrodynamical approach in which the background evolution is governed by a "smoothing" fluid component $(S)$ with equation-of-state parameter

$$
\epsilon \equiv \frac{3}{2}(1+w) \quad \text { with } \quad w \equiv \frac{\rho_{S}}{p_{S}},
$$

where $w$ is the equation of state, $\rho_{S}$ the energy density, and $p_{S}$ the pressure of the smoothing component. Here and throughout the paper we will restrict ourselves to the case that the speed of sound is $c_{s}=1$. (Although it is straightforward to extend the analysis to $c_{s} \neq 1$, current observations require $c_{s}>1 / 3$ [3]; for this range of $c_{s}$, the difference from the $c_{s}=1$ case is nominal.) To have accelerated expansion during the inflationary smoothing phase, the equation-of-state parameter must lie in the range $0<\varepsilon<1$ since the scale factor increases with time as $a \propto t^{1 / \varepsilon}$. To have ultraslow contraction in the ekpyrotic smoothing phase, the analogous condition is $\varepsilon>3$. In both cases, the condition on the equation of state guarantees that, in the Friedmann equation,

$$
H^{2}=\frac{1}{3 M_{\mathrm{Pl}}^{2}}\left(-\frac{3 k}{a^{2}}+\frac{\sigma_{0}^{2}}{a^{6}}+\frac{\rho_{S}}{a^{2 \varepsilon}}+[\text { matter, radiation, etc. }]\right),
$$

the energy density in the smoothing component $\left(\rho_{S} \propto a^{-2 \varepsilon}\right)$ can overtake all other forms of energy density, including matter $\left(\rho \propto a^{-3}\right)$, radiation $\left(\rho \propto a^{-4}\right)$, and gradient energy $\left(\rho \propto a^{-2}\right)$, and can also overtake the anisotropy $\left(\sigma_{0}^{2} / a^{6}\right)$ and spatial curvature $\left(k / a^{2}\right)$. Generally, $\varepsilon \equiv \varepsilon(N)$ is a function of $N$, the number of $e$-folds before the end of the smoothing phase. [Here $M_{\mathrm{Pl}}^{2}=(8 \pi \mathrm{G})^{-1}$ is the reduced Planck mass and $\mathrm{G}$ is Newton's constant.]

In flattening the background with a single fluid of $\varepsilon<1$, inflation also generates a nearly scale-invariant, adiabatic, and Gaussian spectrum for the curvature perturbations on comoving hypersurfaces characterized by a spectral tilt $n_{s}(N)-1[18,19]$, which is also a function of $N$. The same is not true for ekpyrosis. If there is only a single fluid in the contracting phase, the growing-mode, adiabatic perturbations decay and cannot be the seed of structure in the postbang universe [20]. Currently, the best understood way of creating primordial density perturbations is the entropic 
mechanism [15,21]. Here, prebang isocurvature fluctuations are generated by adding a second fluid component; in the simplest case, one that does not affect the background evolution. These isocurvature modes are then converted into density perturbations which source structure in the postbang universe. Another consequence of inflation is the generation of nearly scale-invariant tensor (gravitational wave) fluctuations. The ratio of the tensorto-scalar amplitude as a function of $N$ is labeled $r(N)$. For the ekpyrotic case, the tensor amplitudes are exponentially suppressed compared to inflation and can be considered negligible for the purposes of this discussion. Hence, the detection or nondetection of primordial gravitational waves is a key means of distinguishing the two scenarios.

Assuming only that there was a period of inflation, the point has been made by numerous authors (e.g., see [11] for a recent example) that any observational outcome is possible, rendering the theory unpredictive. The purpose of this paper is to use a hydrodynamical approach to determine how the predictions of inflationary and cyclic cosmologies are affected by the additional assumption that the underlying physics is scale-free. By a scale-free function we mean a power-law form up to a coordinate shift, i.e., $f: \mathbb{R} \rightarrow \mathbb{R}$ is a scale-free function iff there is a coordinate transformation $\pi: \mathbb{R} \rightarrow \mathbb{R}, x \mapsto x+C, C \in \mathbb{R}$, such that

$$
(f \circ \pi)(x)=\beta x^{\alpha}, \quad \alpha, \beta \in \mathbb{R} .
$$

Scale-invariant is the special case where $\alpha=0$.

For our cosmological application, we describe a cosmology as strictly scale-free if both the background equation of state $\varepsilon(N)$ and the perturbations, characterized by $n_{S}(N)-1$ and $r(N)$, are scale-free. We shall show that this condition is highly constraining, leading to specific predictions for $n_{S}-1$ and $r$. In particular, it is immediately apparent from the Friedmann equation, Eq. (2), which can be written as a sum of $a^{-2 \varepsilon_{i}}$, that for a scale-free background the equation-of-state parameter of all components relevant during the smoothing stage must be the same.

Since the case for scale-freeness is based on background evolution and observations on large scales, we also consider background-only scale-freeness in which $\varepsilon$ is precisely scale-free but $n_{S}-1$ can have deviations from scale-freeness on unobservably small length scales $[N=\mathcal{O}(1)]$. In addition, we consider a class of models that weakly break scale-freeness where we analyze deviations in $\varepsilon, n_{S}-1$, and $r$ that only affect unobservably small scales.

\section{INFLATIONARY THEORY}

In order to construct a model with $N^{*} e$-folds of inflation, the following two criteria must be satisfied:
I: sufficient inflation. $-N^{*} e$-folds inflation occur, i.e., $\varepsilon(N)<1$ for $1<N<N_{*}$, and

II: graceful exit.-inflation ends in the last $e$-fold, i.e., $\varepsilon(N=0)=1$; plus $\varepsilon(N>0)<1$ and $\varepsilon(N<0) \geqslant 1$, where $N$ is the number of $e$-folds of inflation remaining until its end $t_{\text {end }}$, defined as

$$
N=\int_{t}^{t_{\text {end }}} H d t
$$

$N=0$ marks the end of inflation. Here, without loss of generality we will assume a single continuous stage of inflation with $N^{*} e$-folds. If these are the only constraints imposed, then $\varepsilon(N)$ can take many forms and the predictions can vary arbitrarily. To transform inflation into a predictive theory, an additional constraint is needed. We use scale-freeness as the added condition.

\section{A. Scale-free inflationary theory}

Scale-freeness, Eq. (3), combined with the two numbered criteria, determines the evolution of $\varepsilon$ during inflation:

$$
\varepsilon(N)=\frac{1}{(N+1)^{\alpha}}, \quad \alpha>0,
$$

where $\alpha$ needs to be strictly positive to satisfy criterion I. That is, the equation of state $\varepsilon(N)$ consistent with the scalefree principle is described by a simple power-law form with a single free parameter, $\alpha$. The second free parameter in Eq. (3), $\beta$, is fixed by criterion II, the condition that $\varepsilon(0)=1$. Considering $\beta$ as a second free parameter, as assumed in Ref. [13], violates criterion II. We will discuss the implications of this restriction below.

To analyze different inflationary solutions, we compute the evolution of the Hubble parameter in terms of $\varepsilon(N)$. Note that we need to assume both criteria I and II for this type of analysis. Here we are being more precise than some previous hydrodynamical treatments. For example, Ref. [12] obtains Eq. (5), but through an inconsistent argument that first assumes $\varepsilon=$ const $\ll 1$ and, hence, violates criterion II. In Ref. [13], $\beta$ is left as a free parameter, which is also inconsistent with criterion II.

For a homogeneous, isotropic, and spatially flat universe, the second Friedmann equation can be written as $\varepsilon=-\dot{H} / H^{2}$. Since $d N=-d \ln a$, we can rewrite the relation as

$$
\varepsilon=\frac{d \ln H}{d N} .
$$

Finally, integration of Eq. (6) together with our expression for $\varepsilon$ in Eq. (5) yields a closed-form expression for $H^{2}$ (or, equivalently, the smoothing energy density $\rho_{S}$ ) as a function of $N$ : 


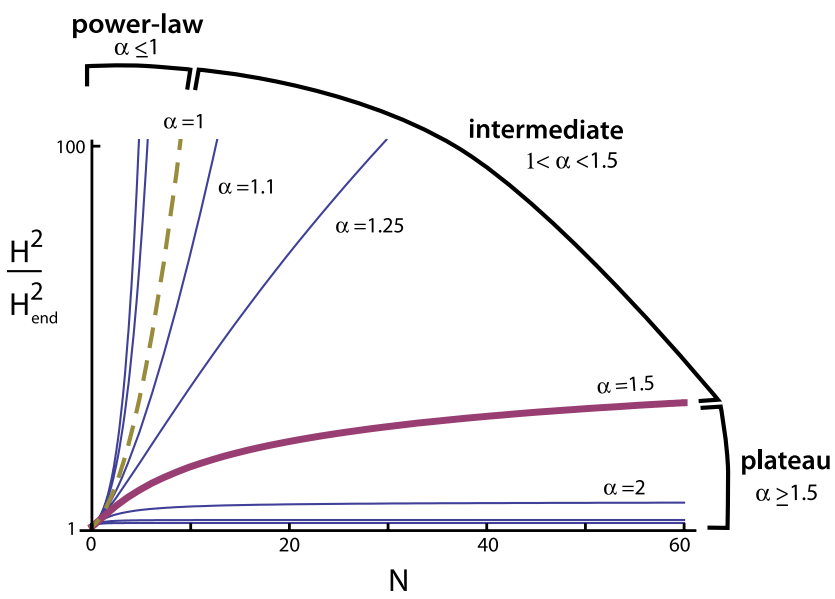

FIG. 1 (color online). In the hydrodynamical picture, scale-free inflationary models can be divided into three classes characterized by $\alpha$ in Eq. (5): the plateaulike class (with $\alpha \geq 1.5$, where $\alpha=1.5$ is the bold thick curve) in which $H^{2}$ flattens out rapidly (well before $N=60$ ) as $N$ increases; the power-law-like class (with $\alpha \leq 1$, where $\alpha=1$ is the dashed curve) in which $H^{2}$ is unbounded above and changes significantly as $N$ increases; and the intermediate class (with $1<\alpha<1.5$ ), which rises like a power law for $N<60$ but which ultimately reaches a plateau at values of $N \gg 60$ that are irrelevant for cosmological predictions. The plateaulike class is most favored by current observations but encounters the problems described in this paper. The power-lawlike models are strongly disfavored by current observations but do not suffer the same problems.

$$
H^{2} / H_{\text {end }}^{2}=\rho_{S} / \rho_{S, \text { end }}=\exp \left[-2 \int_{N}^{0} \varepsilon d N\right]
$$

which reduces in the inflationary case to

$$
H^{2} / H_{\text {end }}^{2}=\left\{\begin{array}{cc}
(N+1)^{2}, & \alpha=1, \\
\exp \left[\frac{2\left(1-(N+1)^{1-\alpha}\right)}{\alpha-1}\right], & \alpha \neq 1,
\end{array}\right.
$$

which is the relevant observable in inflationary dynamics. Note that the Hubble parameter at the end of inflation, $H_{\text {end }}$, is arbitrary.

In Fig. 1 we have plotted $H^{2} / H_{\text {end }}^{2}$ during the inflationary phase as a function of $N$ for different values of $\alpha$. The dashed curve corresponds with the strictly scale-free case, $\alpha=1$. The rest of the curves are background-only scale-free.

The curves divide into three classes: (i) the "plateaulike" class with $\alpha \gtrsim 1.5$ (bold curve) in which $H^{2}$ flattens out and is virtually independent of $N$ over the range $N>60$ (changing by less than 20\%); (ii) the "power-law-like" class with $\alpha \lesssim 1$ in which $H^{2}$ is unbounded above; and (iii) an "intermediate class" with $1<\alpha<1.5$, that appears power-law-like during the last $60 e$-folds (see Fig. 1) but which ultimately reaches a plateau at very large $N \gg 60$ (with $H^{2}$ increasing by more than $20 \%$ for $N>60$ ). ${ }^{1}$

The expression for the equation-of-state parameter as defined in Eq. (5) enables us to derive predictions for the spectral tilt and the tensor-to-scalar ratio of primordial density perturbations. Since $\varepsilon(N)$ does not change rapidly, i.e.,

$$
\frac{d \ln \varepsilon}{d N}=-\frac{\alpha}{N+1}, \quad \frac{d^{2} \ln \varepsilon}{d N^{2}}=\frac{\alpha}{(N+1)^{2}} \lesssim \mathcal{O}(1),
$$

we can use the approximation [23]

$$
n_{S}-1 \approx-2 \varepsilon+\frac{d \ln \varepsilon}{d N} .
$$

Substituting $\varepsilon$ from Eq. (5) yields

$$
n_{S}-1 \approx-\frac{2}{(N+1)^{\alpha}}-\frac{\alpha}{N+1}
$$

It is instructive to note that $n_{S}-1$ has a maximum value of

$$
\begin{aligned}
\left(n_{S}-1\right)\left(\alpha_{0}\right) & =-\frac{\ln [2(N+1) \ln (N+1)]+1}{(N+1) \ln (N+1)} \\
\text { for } \quad \alpha_{0} & =\frac{\ln [2(N+1) \ln (N+1)]}{\ln (N+1)} .
\end{aligned}
$$

For example, with $N=60$, we have $\alpha_{0} \simeq 1.5$ and $\left(n_{S}-1\right)\left(\alpha_{0}\right) \simeq-0.03$. This red tilt is the minimum deviation from Harrison-Zel'dovich-Peebles spectrum (HZP) for a scale-free inflationary model and is close to the observed value. (Without scale-freeness or criterion II, $n_{S}$ can be arbitrarily close to HZP or yield a blue tilt.) This extremum lies almost precisely at the borderline between the intermediate and plateaulike classes. (The extremum is described as being at $\alpha \approx 2$ in [13], but, in our analysis, this crude approximation would give the wrong impression that it corresponds to the observationally favored models deep in the plateau range when it actually corresponds to a disfavored case.)

Finally, with the standard normalization, the tensor-toscalar ratio is [12]

$$
r \approx 16 \varepsilon=\frac{16}{(N+1)^{\alpha}}
$$

\section{B. Cosmological problems}

The plateaulike hydrodynamical class, especially near $\alpha=2$, is the one favored by current observations [2], yet it suffers from a series of problems, some of which are analogous to those described in the analysis of scalar field

\footnotetext{
${ }^{1}$ Note that "intermediate" here refers to the range of scale-free models that have a mix of characteristics between plateau and power-law scale-free behavior. This is distinct from Ref. [22], where "intermediate" refers to cases where the scale factor $a(t) \propto \exp \left(A t^{f}\right)$, which is not scale-free and so does not fit into our classification.
} 
potentials in [14] and some of which have not been discussed previously.

(i) Extra parameters. - The plateaulike class has the property that $H^{2}$ is nearly flat except for the last $e$-fold or so when the expansion rate suddenly decreases; see the feature at small $N$ in the plateaulike curves in Fig. 1. That means whatever microphysics accounts for $\varepsilon(N)$ must have an extra parameter and/or field compared to the power-law-like models adjusted to rapidly cutoff the inflation after a long period of a nearly constant $H^{2}$. We will see this effect in Sec. V when we translate our hydrodynamical results into models of scalar fields and inflaton potentials.

(ii) Hydrodynamical initial conditions problem.-As originally imagined, inflation was supposed to smooth and flatten the universe beginning from arbitrary initial conditions after the big bang [8]. However, this view had to be abandoned as it was realized that large inflaton kinetic energy and gradients prevent inflation from starting.(Inflation is an attractor for some potentials [24], provided the deviations from inflation are small, but the basin of attraction is small compared to typical initial conditions [25] considered below.) Consequently, inflation can only take hold if the entropy, kinetic energy, and gradients within a Hubble-sized patch is exceedingly small.

We note that the later that inflation starts, the greater is the physical size of a Hubble patch and the more unlikely is the initial condition. A distinctive feature of the power-law-like hydrodynamic class $(\alpha \leq 1)$ is that $H^{2}$ is unbounded above. Hence, inflation can begin, in principle, at arbitrarily high $H^{2}$ or, equivalently, over a small patch where the initial conditions are less unlikely compared to cases where inflation starts later. This includes inflation beginning immediately after the big bang when the energy density is at the Planck scale. By contrast, inflation for models in which $H^{2}$ is bounded above (i.e., all $\alpha>1$ ) can only begin after the universe expands enough for the energy density to drop to the level of the plateau, $M_{\mathrm{I}}^{4}$. The Planck2013 constraint on $r\left(r_{0.002}<0.12\right.$ at $95 \%$ C.L. $)$ [2] yields

$$
M_{\mathrm{I}}^{4} \lesssim \frac{3 \pi^{2} A_{s}}{2} r M_{\mathrm{Pl}}^{4} \sim 10^{-12} M_{\mathrm{Pl}}^{4} \frac{r_{*}}{0.12}
$$

at $95 \%$ C.L., where $A_{s}$ is the scalar amplitude and $r_{*}$ the value of $r$ evaluated at Hubble exit during inflation of mode with wave number $k_{*}$. This is well below the Planck density at a time when the Hubble volume is, by simple comparison of the scales $M_{\mathrm{Pl}} / M_{\mathrm{I}} \sim 10^{3} \times\left(10^{16} \mathrm{GeV} / M_{\mathrm{I}}\right), 10^{9}$ times (or more) greater [14]. In this case, some combination of gradient energy density, spatial curvature, and radiation must necessarily dominate immediately after the big bang and for a substantial period thereafter before inflation can ever take hold. A well-known problem, though, is that gradient energy and spatial curvature tend to block inflation by causing regions of space to collapse before inflation can start [14]. That is, inflation can only begin for the plateaulike models if there is the extraordinary additional assumption that the universe emerges from the big bang with a patch:

$$
\begin{aligned}
R^{3}\left(t_{\mathrm{Pl}}\right) & \gtrsim\left[a\left(t_{\mathrm{Pl}}\right) \int_{t_{\mathrm{Pl}}}^{t_{\mathrm{I}}} \frac{d t}{a}\right]^{3} \sim\left[\frac{a\left(t_{\mathrm{Pl}}\right) H\left(t_{\mathrm{Pl}}\right)}{a\left(t_{\mathrm{I}}\right) H\left(t_{\mathrm{I}}\right)} H^{-1}\left(t_{\mathrm{Pl}}\right)\right]^{3} \\
& >10^{9}\left(\frac{10^{16} \mathrm{GeV}}{M_{\mathrm{I}}}\right)^{3} H^{-3}\left(t_{\mathrm{Pl}}\right)
\end{aligned}
$$

that is smooth and flat on scales $10^{9}$ times greater than required for the unbounded power-law-like case [26]. Our hydrodynamic analysis divides the inflationary models along the dashed line $(\alpha=1)$ in Fig. 1 between those that require this extraordinary assumption (plateaulike and intermediate with $\alpha>1$ ) and those that do not $(\alpha \leq 1)$.

(iii) Hydrodynamical unlikeliness problem.-Even assuming the rare initial conditions are satisfied, the observationally favored plateaulike models $(\alpha \approx 2)$ produce exponentially less smooth and flat volume than the power-law-like or intermediate class models with $1 \lesssim \alpha<1.5$. This leads to the hydrodynamic version of the "unlikeliness problem" similar to (but not identical to; see Sec. V) the one discussed in [14]: First, let us imagine a complex energy landscape in which there are many different kinds of paths corresponding to different a mix of power-law, intermediate and plateaulike classes that proceed to the same vacuum. The most likely path is the one that produces the most number of $e$-folds of inflation.

For each $\alpha$, we can compute the largest value of $N$ for which the density fluctuation $\delta \rho / \rho(N)$ is less than 1 . For larger $N$ where $\delta \rho / \rho$ exceeds 1 , quantum fluctuations totally spoil the homogeneity and curvature. Hence, $N_{\max }(\alpha)$, the maximum number of $e$-folds as a function of $\alpha$, is determined by the condition

$$
\delta \rho / \rho\left(N_{\max }\right)=1 .
$$

The fluctuation amplitude is

$$
\delta \rho / \rho(N) \simeq \frac{H(N)}{M_{\mathrm{Pl}} \sqrt{\varepsilon(N)}}
$$

(for the derivation use, for example, $\delta \rho / \rho=H / \dot{\phi}$ and $\left.\dot{\phi}^{2}=\rho+p\right)$. Substituting the expressions we previously found for $H^{2}$ and $\varepsilon$, Eqs. (16) and (17) together give

$$
N_{\max }(\alpha)=-1+\left(\frac{1}{2} \alpha W(z)\right)^{\frac{1}{1-\alpha}}
$$




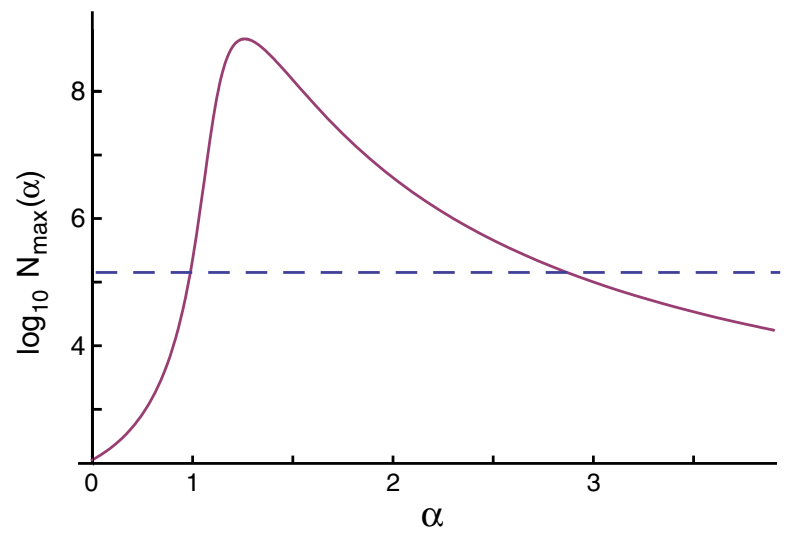

FIG. 2 (color online). A logarithmic plot of the maximum number of $e$-folds $N_{\max }(\alpha)$ for scale-free models as a function of the hydrodynamic variable $\alpha$. The plot assumes initial conditions can be set perfectly smoothly in the initial Hubble patch.

where $W$ is the Lambert $W$ function, and its parameter

$$
z=\frac{2}{\alpha}\left(10^{5} \cdot 61^{\alpha / 2} \cdot \exp \left(\frac{61^{1-\alpha}}{1-\alpha}\right)\right)^{\frac{2}{\alpha}(1-\alpha)},
$$

and $\delta \rho / \rho$ is normalized such that $\delta \rho / \rho(N=60)=$ $10^{-5}$. For $\alpha=1, N_{\max }(\alpha)$ is $61 \times 10^{10 / 3} \approx 10^{5}$.

As illustrated in Fig. 2, $N_{\max }$ is maximal overall for $\alpha \simeq 1.25$; among the power-law-like cases, $\alpha=1$ is most favored; and among the plateaulike models $\alpha=$ 1.5 is most favored. The differences in inflated volume in each case are exponentially large, of order $\exp \left(10^{5-8}\right)$, so "favored" means "very strongly favored" [2]. Note that $\alpha=2$ is strongly disfavored; yet, this is the inflationary type model that is currently most favored observationally.

These estimates for $N_{\max }(\alpha)$ are, however, based on the idea of a complex energy landscape with many different types of paths to each minimum, assuming that the initial conditions when the universe emerged from the big bang could be set with arbitrary accuracy so that the energy density in the smoothing component is the maximum possible, $3 H^{2}\left(N_{\max }(\alpha)\right)$ in Planck units. However, a more serious problem that applies for even simple energy landscapes is that most patches of space are likely to have large gradient energy that will spoil inflation altogether. Even if we eliminate those patches and consider only homogeneous patches, in each patch there remain different mixes of radiation, kinetic energy, potential energy, and other forms of energy such that, typically, we do not have patches at precisely the ideal potential energy to obtain $N_{\max }$. Hence we should imagine some flex of order $x$ in the amount of the initial potential energy. A reduction of the average energy density in the patch by a factor $x$ requires a revised estimate $N_{\max }(\alpha, x)$ :

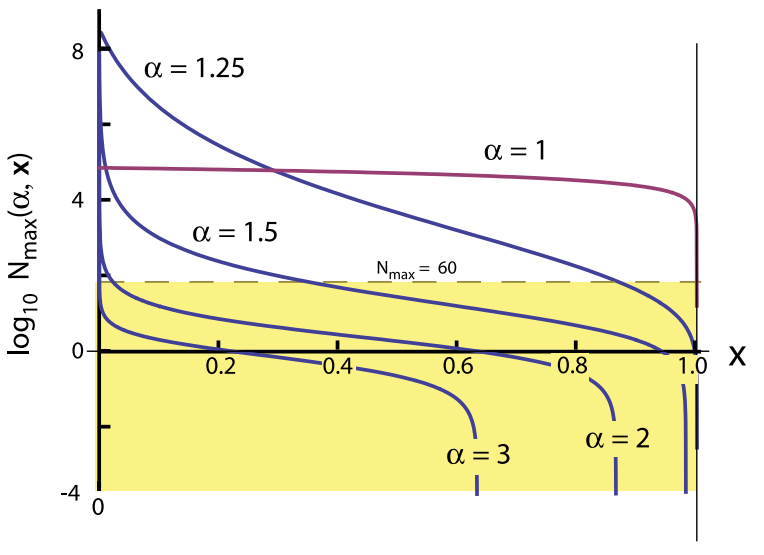

FIG. 3 (color online). The sensitivity of $N_{\max }$ to the initial energy density in the smoothing component at the Planck time when the universe first emerges from the big bang. If the energy density in a patch could be set with perfect precision, the maximum number of $e$-folds of inflation would be $N_{\max }(\alpha) \equiv$ $N_{\max }(\alpha, 0)$ plotted in Fig. 2. Because of contributions of other forms of energy (kinetic energy, radiation energy, etc.), we assume a variation of $x$ percent from perfect precision and compute how this affects the maximum number of $e$-folds, $N_{\max }(\alpha, x)$, as shown in the logarithmic plot above. Note that the $N_{\max }(\alpha)$ in Fig. 2 is equal to $N_{\max }(\alpha, 0)$. The plot shows that $N_{\text {max }}(\alpha, x)$ for $\alpha=1$ (strictly scale-free power-law-like models) is rather insensitive to $x$. By contrast, plateaulike models $(\alpha \geq 1.5)$ are so extremely sensitive to $x$ that, unless the initial energy density of the smoothing component is set with extraordinary precision, the value of $N_{\max }(\alpha, x)$ is much less than that for the power-law-like class and less than the minimal 60 needed for inflation. The shaded region corresponds to insufficient inflation.

$$
N_{\max }(\alpha, x)=\left(N_{\max }(\alpha, 0)^{1-\alpha}-\frac{\alpha-1}{2} \ln (x)\right)^{\frac{1}{1-\alpha}}-1,
$$

which equals $61 \times 10^{10 / 3} \sqrt{x}$ for $\alpha=1$. Because plateaulike models with $\alpha \geq 1.5$ are so flat for large $N$, a reduction in average $H^{2}$ by some factor $x$ produces a much greater reduction in $N_{\max }(\alpha, x)$ relative to $N_{\max }(\alpha) \equiv N_{\max }(\alpha, 0)$ than is found for power-law-like models.

Figure 3 shows $\log N_{\max }$ as a function of $x$ for different values of $\alpha$. The dashed line corresponds to the strictly scale-free, unbounded power-law-like case with $\alpha=1$; the thin black curves correspond to models with $\alpha$ values of $1.25,1.5,2$, and 3 ; the red horizontal line marks $60 e$-folds. It is clear that the plateaulike models fail to reach $N=60 e$-folds for even a small $x$, while the power-law-like models and intermediate class models are comparatively insensitive to the initial distribution of energy in the patch. In sum, there are three classes of scale-free inflationary scenarios. Power-law-like models $(\alpha \leq 1)$ do not suffer from the initial conditions problem or unlikeliness problem. Models of the intermediate 
class have the initial conditions problem, but not the unlikeliness problem. However, these models are all observationally disfavored currently [2]. The observationally favored plateaulike models with $\alpha=2$ suffer from all the problems described above. Hence, the theoretically favored scale-free inflationary models are observationally disfavored and vice versa. The fact that the initial conditions and unlikeliness problems impose different constraints illustrates that they are logically distinct, a point that some have disputed in discussions of [14].

\section{Deviations from scale-freeness}

We have thus far considered $\epsilon(N)$ that have a scale-free form. The case $\alpha=1$ is strictly scale-free in that the functions that describe the background, $\epsilon(N)$ and $H(N)$, as well as the functions that describe the perturbations

$$
n_{S}(N)-1=-\frac{3}{N+1}
$$

are all simple power laws (or power laws with shifts).

For $\alpha \neq 1$, the background functions are still scale-free but the spectral index is not:

$$
n_{S}(N)-1=-\frac{2}{(N+1)^{\alpha}}-\frac{\alpha}{N+1},
$$

so there is only background scale-freeness.

For weakly broken scale-freeness, there can be no complete treatment since "weakly" is an imprecise term. Here we consider in this category deviations from scalefreeness at the background level but only on length scales that are unobservably small (corresponding to small $N$ ):

$$
\epsilon=\frac{\beta}{(N+1)^{\alpha}}-\frac{\beta-1}{(N+1)^{\alpha+\gamma}}, \quad \text { with } \quad \beta, \gamma>0, \beta \neq 1,
$$

where this form is designed to still satisfy inflationary criteria I and II. For the deviation to be small, in addition, it is necessary that

$$
|1-1 / \beta| \ll(N+1)^{\gamma} \text { and }|\beta-1|<1
$$

for observable $N$. Then, with an additional free parameter, the predictions are modified:

$$
\begin{aligned}
\epsilon \approx & \frac{\beta}{(N+1)^{\alpha}}, \\
n_{S}-1 & \approx \begin{cases}-\frac{2 \beta}{(N+1)^{\alpha}}, & \alpha<1, \\
-\frac{2 \beta+1}{(N+1)}, & \alpha=1, \quad r \approx \frac{16 \beta}{(N+1)^{\alpha}} . \\
-\frac{\alpha}{N+1}, & \alpha>1,\end{cases}
\end{aligned}
$$

As we shall discuss below in Sec. V, the case $\alpha=1$ is of particular interest as it corresponds to power-law inflaton $(\phi)$ potentials $V(\phi) \propto \phi^{n}$ with $n=4 \beta$. From Eq. (25), we note that the weakly scale-free breaking inflationary models $(\beta \neq 0)$ entail two independent parameters while strictly scale-free inflationary theory involves exactly one free parameter.

\section{CYCLIC THEORY}

In the following section, we carry out the same type of hydrodynamical analysis for the cyclic theory that we previously did for inflation. In order to construct a model with $\mathcal{N}^{*} e$-folds of ultraslow contraction (ekpyrosis) that flattens and smoothes the universe, the two criteria analogous to those used for inflation are as follows:

$\mathrm{I}^{\prime}$ : sufficient ekpyrosis. $\mathcal{N}^{*} e$-folds of ekpyrosis occur, i.e., $\epsilon(\mathcal{N})>3$ for $1<\mathcal{N}<\mathcal{N}^{*}$; and

II': exit.-ekpyrosis ends in the last $e$-fold, i.e., $\epsilon(\mathcal{N}>0)>3$, and $\epsilon(0)=3$.

We have introduced the dimensionless time variable $\mathcal{N}$, defined by

$$
\mathcal{N} \equiv \ln \left(\frac{a_{\text {end }} H_{\text {end }}}{a H}\right)
$$

$\mathcal{N}$ measures the number of $e$-folds of modes that exit the horizon before the end of ekpyrosis. It is related to the time variable $N$ used in the previous section by $d \mathcal{N}=(\epsilon-1) d N$. For inflation $\mathcal{N} \approx N$, since $H \approx$ const during accelerated expansion. For ekpyrosis, on the other hand, $\mathcal{N} \gg N$ because $H$ grows significantly during ultraslow contraction while $a$ shrinks very slowly, so $\mathcal{N}$ is the correct time variable to use. Here, in analogy with the treatment of inflation, we assume a single continuous stage of ekpyrosis with $\mathcal{N}^{*} e$-folds.

\section{A. Scale-free cyclic theory}

Scale-freeness, combined with these two criteria, determines the evolution of $\epsilon$ during the ekpyrotic phase. From Eq. (3) together with criteria I/ and II/, we have

$$
\epsilon(\mathcal{N})=3(\mathcal{N}+1)^{\alpha_{1}}, \quad \alpha_{1}>0 .
$$

That means the shape of the equation-of-state parameter consistent with the scale-free principle is a simple powerlaw form with a single free parameter. The second free 


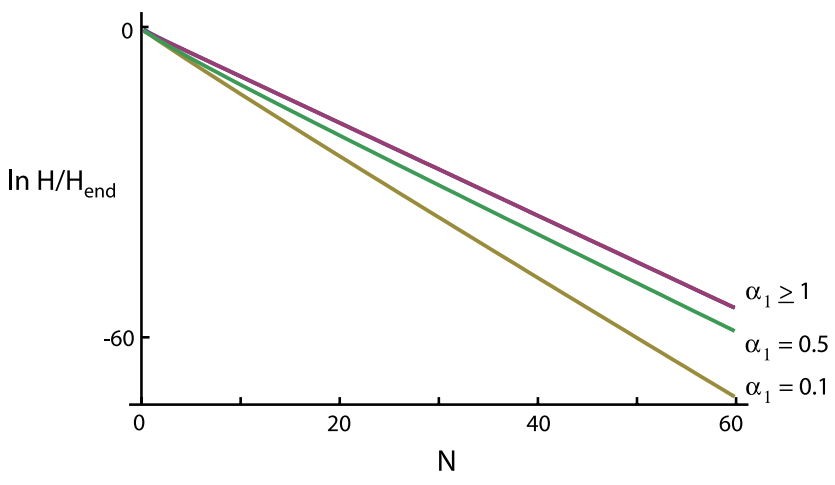

FIG. 4 (color online). Plot of $\ln H^{2} / H_{\text {end }}^{2}$ vs $\mathcal{N}$ for the cyclic picture for a range of $\alpha_{1}$.

parameter, $\beta_{1}$, in Eq. (3) is fixed by criterion $\mathrm{II}^{\prime}$, which requires $\epsilon(0)=3$.

To analyze different cyclic solutions, we study the evolution of the total energy density $H^{2} / H_{\text {end }}^{2}$ during ekpyrosis. Substituting Eq. (27) into Eq. (7) yields

$$
H^{2} / H_{\text {end }}^{2}=\exp \left(-2 \mathcal{N}+2 \int_{\mathcal{N}}^{0} \frac{d \mathcal{N}}{3(\mathcal{N}+1)^{\alpha_{1}}-1}\right)
$$

Note that this expression reflects a characteristic feature of an ekpyrotic phase that $H^{2}$ grows by many orders of magnitude during smoothing. Figure 4 shows a logarithmic plot of $H^{2} / H_{\text {end }}^{2}$ for the ekpyrotic phase as a function of $\mathcal{N}$ for different values of $\alpha_{1}$.

In contrast to inflation, cyclic models do not divide into different classes. In fact, for $\alpha_{1} \gtrsim 1$ all of the $H^{2}$ curves lie virtually on top of one another such that the Hubble parameter proves effectively independent of $\alpha_{1}$. Hence, the unlikeliness problem, based on comparing the probability of different classes, cannot arise for the cyclic theory. In addition, it follows from the $\alpha_{1}$ independence that choosing a value of $\alpha_{1}$ to fit observational data does not involve any special fine-tuning relative to the general class of models.

The initial conditions requirement is extremely mild. It suffices to have a volume of space on the scale of meters in diameter that is absent of black holes or nonlinear structure at the beginning of the contraction phase [27]. The ekpyrotic mechanism will smooth and flatten this region and the bounce will transform this region during the expansion phase into a size of order the Hubble volume today. The initial condition can be reached in a number of ways, including by having an expanding phase precede the contraction phase. For example, in the cyclic scenario, the initial condition is easily achieved by having the ekpyrotic phase preceded by an expanding dark energy dominated phase just like the current phase of our Universe. Consider that the present Universe already contains exponentially many patches that satisfy the initial condition requirements and any further expansion only increases their number.
Having an expanding dark energy phase turn into a contracting phase is known to be quite straightforward to achieve, e.g., by having a scalar field roll or tunnel from a phase with positive potential density to a phase with a negative potential energy density [28,29]. In order for ekpyrosis to occur, no further criteria need to be satisfied; expansion can turn into contraction at arbitrarily low energies for an $\alpha_{1}$ since there is no (classical) limit in Fig. 4 on how low $H$ can be when contraction begins for any $\alpha_{1}$ (so the choice of $\alpha_{1}$ does not require extra tuning). By contrast, for inflation, assuming an expanding phase after the bang is not sufficient since the natural conditions after the bang would have large gradient and kinetic energies that would block the initiation of inflation.

In sum, at background level, none of the problems pointed out above for inflation arise for the cyclic model. There is no fine-tuning or unlikeliness problem, and there is no initial conditions problem comparable to the inflationary case.

At the perturbative level there is a notable conceptual difference between inflation and the cyclic model, at least according to most current versions of cyclic theory. Namely, the generation of primordial density perturbations is assumed to be a two-stage process. First, entropy or isocurvature perturbations are created before the bounce. These perturbations are then converted into primordial density perturbations at some time during the transition from big crunch to big bang [15].

Modeling this scenario in a hydrodynamical approach requires a two-component fluid: one fluid component governs the background evolution and the other is responsible for the generation of isocurvature fluctuations. The background fluid component can be described by an equation-of-state parameter, $\epsilon_{1}(\mathcal{N})$, as defined in Eq. (27):

$$
\epsilon_{1}(\mathcal{N})=3 \beta_{1}(\mathcal{N}+1)^{\alpha_{1}}, \quad \alpha_{1}>0,
$$

where $\beta_{1}=1$ according to criterion II. The equation-ofstate parameter for the second fluid, $\epsilon_{2}(\mathcal{N})$, must also satisfy the requirement of scale-freeness. Hence, from Eq. (3), it is necessary (but not sufficient, as we point out below) for $\epsilon_{2}(\mathcal{N})$ to take the form

$$
\epsilon_{2}(\mathcal{N})=3 \beta_{2}(\mathcal{N}+1)^{\alpha_{2}}, \quad \alpha_{2} \in \mathbb{R} .
$$

If this component satisfies the null energy condition, $\beta_{2}$ must be greater than or equal to zero.

Before imposing any further conditions, the general expression for the spectral tilt of density perturbations is

$$
\begin{aligned}
n_{S}(\mathcal{N})-1= & 3-\sqrt{1+8 \kappa} \\
& \times\left(1+3 \cdot \frac{1-2 \kappa}{1+8 \kappa} \cdot \frac{2}{\epsilon_{1}}+\frac{8-5 \kappa}{1+8 \kappa} \cdot \frac{\epsilon_{1}, \mathcal{N}}{\epsilon_{1}}\right),
\end{aligned}
$$

where 


$$
\kappa(\mathcal{N})=\epsilon_{2} / \epsilon_{1}=\left(\beta_{2} / \beta_{1}\right)(\mathcal{N}+1)^{\alpha_{2}-\alpha_{1}}
$$

(see the Appendix for the derivation). In the limit of constant $\kappa(N) \approx 1$, the expression reduces to

$$
n_{S}-1=\frac{2}{\epsilon_{1}}-\frac{\epsilon_{1}, \mathcal{N}}{\epsilon_{1}}+\frac{4}{3}(1-\kappa),
$$

in agreement with $[21,30]$.

\section{B. Deviations from scale-freeness}

For the strictly scale-free case, both the background and the perturbations must be simple power laws. For the background Friedmann equations, we mean that the dominant contribution to $H^{2}$ in Eq. (2) should be a simple power law in $a$. As noted above in Eq. (3), this requires $\epsilon_{1}(\mathcal{N})=$ $\epsilon_{2}(\mathcal{N})$ with $\alpha_{1}=\alpha_{2}=1$ and $\beta_{1}=\beta_{2}$. Then, the prediction for the spectral tilt is

$$
n_{S}-1=\frac{2}{\epsilon_{1}}-\frac{\epsilon_{1}, \mathcal{N}}{\epsilon_{1}}=-\frac{1}{3(\mathcal{N}+1)} .
$$

For the background-only scale-free case, we still require $\beta_{2}=\beta_{1}=1$ and $\alpha_{1}=\alpha_{2}$, but the $\alpha$ 's need not be 1 . Then, the spectral tilt has a small deviation from scale-freeness:

$$
n_{S}-1=\frac{2}{\epsilon_{1}}-\frac{\epsilon_{1}, \mathcal{N}}{\epsilon_{1}}=\frac{2}{3(\mathcal{N}+1)^{\alpha_{1}}}-\frac{\alpha_{1}}{\mathcal{N}+1},
$$

in agreement with [15,21]. Note that, even though there are two fluid components, the expression for $n_{s}$ has only one free parameter, as in the case of inflation.

Finally, we consider the weakly broken scale-free case in which deviations from scale-freeness occur only on unobservable scales. As with inflation, there is no absolute definition of weakly broken scale-free, but we consider two types of deviations that arise in microphysical models of scalar fields.

First, a very weakly broken scale-free background occurs if $\beta_{2}$ is close to but not equal to $\beta_{1}=1$, or, equivalently, $0<|\kappa-1| \ll 1$. In this case, the expression for the tilt reduces to the simpler form

$$
\begin{aligned}
n_{S}-1 & =\frac{4}{3}(1-\kappa)+\frac{2}{\epsilon_{1}}-\frac{\epsilon_{1}, \mathcal{N}}{\epsilon_{1}} \\
& =\frac{4}{3}(1-\kappa)+\frac{2}{3(\mathcal{N}+1)^{\alpha_{1}}}-\frac{\alpha_{1}}{\mathcal{N}+1},
\end{aligned}
$$

in agreement with $[21,30]$. A second type of deviation from background scale-freeness is to choose $\beta_{1} \neq 1$, which generates additional contributions to $n_{S}$ analogous to the inflationary case; see Eqs. (23) and (25). As with the background case, the weakly broken scale-free case for the two-fluid-component cyclic scenario has the same number of free parameters as for inflation, so neither theory is advantageous by this measure.

\section{SCALE-FREE SCALAR FIELDS AND POTENTIALS}

The problems we identified for inflationary theory are similar but not identical to the issues identified previously in [14], using a model-dependent analysis based on assuming that inflation is driven by scalar fields with specific potential forms. In order to compare the two approaches, we translate our hydrodynamical scale-free models into the field picture, first for inflation and subsequently for cyclic cosmology.

\section{A. Scale-free inflationary potentials}

The construction of scale-free inflationary potentials corresponding to the hydrodynamical models described in previous sections is based on assuming single-field, slow-roll inflation with canonical kinetic energy density and $\rho_{S} \simeq V(\phi)$, where $V(\phi)$ is the potential energy density for the inflaton scalar field $\phi$. Following the method presented in [13], the Friedmann equations together with the identity $\dot{\phi}^{2}=\rho_{S}+p_{S}$ yield

$$
\begin{aligned}
\frac{\phi-\phi_{\mathrm{end}}}{M_{\mathrm{Pl}}} & = \pm \int_{N}^{0} \sqrt{2 \epsilon} d N \\
& = \pm \sqrt{2} \cdot\left\{\begin{array}{cc}
-\ln (N+1), & \alpha=2 \\
\frac{2}{2-\alpha}\left(1-(N+1)^{\frac{2-\alpha}{2}}\right), & \text { otherwise. }
\end{array}\right.
\end{aligned}
$$

Then, with Eq. (7) we find the expression for the inflationary potential

$$
V(\varphi)=\left\{\begin{array}{cc}
\lambda \varphi^{4}, & \alpha=1, \\
V_{\text {end }} \exp \left[2-2 \exp \left(-\frac{\varphi-\varphi_{\text {end }}}{\sqrt{2} M_{\mathrm{Pl}}}\right)\right], & \alpha=2, \\
V_{\text {end }}\left(3-(N(\varphi)+1)^{-\alpha}\right) \exp \left[\frac{2}{1-\alpha}\left((N(\varphi)+1)^{1-\alpha}-1\right)\right], & \text { otherwise }
\end{array}\right.
$$

where $N(\phi)$ is given by Eq. (37). 
In the hydrodynamical analysis, we found that the scale-free inflationary models divide into three classes: power-law-like $(\alpha \leq 1)$, intermediate $(1<\alpha<1.5)$ and plateaulike $(1.5 \leq \alpha)$. In the scalar-field potential analysis, the first class, the power-law-like models, divides into two cases: the strictly scale-free $\alpha=1$ case, corresponding to $V(\phi)=\lambda \phi^{4}$ with only a single dimensionless parameter; and $\alpha<1$, for which the potential is exponential with a power-law prefactor and a dimensionful parameter. Both cases are free of the hydrodynamical initial conditions and unlikeliness problems described here and the corresponding problems described for potentials in [14]. However, in the latter case $(\alpha<1)$, graceful exit occurs since the powerlaw prefactor becomes significant in the last $e$-fold, adding a feature to the exponential potential. The added feature breaks the appealing scale-free character. Hence, the scalar field potential analysis picks out the $\alpha=1$ strictly scalefree case as being simplest among the power-law-like class.

The intermediate class of hydrodynamical models $(1<\alpha<1.5)$ translates into plateau potentials with large-field inflation. Unlike the $\alpha=1$ case, these models require tuning one or more dimensionful parameters to satisfy cosmological constraints on the number of $e$-folds and the density fluctuation amplitude, $\delta \rho / \rho \sim 10^{-5}$. As in the hydrodynamical analysis, the predictions for $n_{s}-1$ and $r$ during the last $60 e$-folds depend on the shape of the potential beyond the very flat part of the plateau as the potential dips sharply towards zero. Consequently, the predictions are very similar to expectations for monomial potentials, such as $V(\phi) \sim m^{2} \phi^{2}$. However, because the potentials are plateaulike at large $\phi$, these models exhibit the initial conditions problem described here and in [14].

Finally, the plateaulike class of hydrodynamic models are split into two cases when translated into scalar fields and potentials. For $1.5 \leq \alpha \leq 2$, they correspond to largefield models and include Higgs [31] (with action expressed in the Einstein frame). They exhibit the initial conditions and unlikeliness problems and require tuning one or more dimensionful parameters to satisfy cosmological constraints. For $\alpha>2$, the potentials correspond to small-field plateau potentials such as new inflation $[9,10]$ which exhibit the initial conditions and unlikeliness problems and require two or more dimensionful parameters $V_{\text {end }}$ and $\phi_{\text {end }}$ to yield the correct spectrum of primordial density fluctuations and sufficient $e$-folds of inflation.

In sum, the model-dependent analysis based on inflaton fields and potentials gives a somewhat different view of the landscape of scale-free inflationary models and their problems but on the whole confirms and sharpens the results of the hydrodynamic analysis. From either point of view, the strictly scale-free $\alpha=1$ case is the least problematic among all the models and all classes. The analysis based on scalar fields with scale-free potentials splits two of the hydrodynamic classes into two distinct subgroups through the conversion from $N$ to $\phi$ as the independent variable. It further suggests a hierarchy from least to most problematic, where the least problematic and requiring the least dimensionful parameters is the strictly scale-free $\alpha=1$ model followed by the power-law-like models with $\alpha<1$ and intermediate class models. Unfortunately, the inflationary models favored by present data do not belong to either of these groups. The results also show that, in the plateaulike class, large-field models with $\alpha<2$ require fewer dimensionful parameters than small-field models $(\alpha>2)$.

We note that the hydrodynamic unlikeliness problem described in this paper is more general than the version identified in [14]. In [14] it was shown specifically for small-field plateaulike models that inflation is exponentially less likely in a generic energy landscape than monomial potentials $V \sim \phi^{n}$. The results in the present paper based on scale-freeness show that the entire plateaulike class is theoretically disfavored compared to the entire power-law-like class, whether small-field or large-field inflation.

Among monomial inflationary potentials $V \sim \phi^{n}$, the only strictly or background-only scale-free example is the conformally invariant case, $n=4$, corresponding to $\alpha=1$, which we have shown is the least problematic. ${ }^{2}$ Recovering other power-law potentials requires explicitly breaking scale-freeness while still respecting the inflationary conditions, criteria I and II. For example, by introducing two additional nonzero parameters $\beta$ and $\gamma$ as defined in Eq. (23), the equation-of-state parameter can be made to follow closely the equation of state that can be obtained for $n=4 \beta$. Note that $\phi^{2}$ requires non-negligible scale-free breaking in the sense that $\beta$ is significantly less than one. Power-law models with yet smaller powers, such as $\phi^{2 / 3}$, require even greater deviations from scale-freeness.

However, introducing this extra scale-freeness breaking degree of freedom could be a dangerous course. There already exists a spectrum of inflationary cases parameterized by $\alpha$ in the background scale-free limit. Having a spectrum of cases reduces the predictive power of the paradigm. Applying the same scale-free breaking degree of freedom, $\beta$, for all $\alpha$ further broadens the range of possibilities and increases the number of parameters. This reduces the predictability to the point where there can be more parameters than observational constraints. Furthermore, the breaking of scale-freeness only complicates the model without resolving any of the problems identified for the scale-free cases. Given that the Universe seems so simple based on observations, it is problematic to consider cases with more parameters than the inflationary paradigm requires or the data can constrain.

\footnotetext{
${ }^{2}$ Here we correct the crude approximation made in [13] which led to the incorrect conclusion that $\phi^{6}$ is the strictly scale-free solution.
} 
Not everyone would agree with this assessment. In order to address the initial conditions problem described by Ijjas, Steinhardt, and Loeb [14] and in this paper, the authors have introduced potentials with double inflation, first a power-law-like phase and then a plateaulike phase $[11,32,33]$; or they have introduced an energy landscape with false vacuum inflation tunneling to a plateau [34]. In these cases, the deviation from scale-freeness is intentionally designed to occur for modes outside the Hubble horizon beyond the range of observational tests. From a theoretical perspective, the logic is odd: if the physics underlying inflation is not truly scale-free, why should the deviation from scale-freeness only show up on unobservably large scales? The only purpose is to evade the initial conditions problem while remaining consistent with observations. But the cost is too precious. As evidenced by the example of Ferrara et al. [11], this approach introduces enough new parameters and enough tuning that any outcome for $n_{S}-1$ and $r$ becomes possible, such that inflationary cosmology loses all predictive power.

\section{B. Scale-free cyclic potentials}

As explained in the Appendix, a generic form for the scalar-field potential energy density in the cyclic model can be cast in the form

$$
V(\sigma, s)=V(\sigma, 0)\left(1+\frac{1}{2} \kappa \frac{V,_{\sigma \sigma}}{V(\sigma, 0)} s^{2}+\mathcal{O}\left(s^{3}\right)\right),
$$

where $\sigma$ corresponds with the fluid component governing the background evolution described by $\epsilon_{1}$ and $s$ is the field representation of the fluid with equation-of-state parameter $\epsilon_{2}$ that generates the isocurvature fluctuations before the bounce (that are converted to the nearly scale-invariant curvature perturbations during the bounce). The background evolution is along the $\sigma$ direction with $s=0$. The parameter $\kappa$ is the ratio $\epsilon_{2} / \epsilon_{1}$ defined in Eq. (32), which relates the curvature of the potential energy density along the $s$ direction to the curvature along the $\sigma$ direction. The strictly scale-free case corresponds to $\kappa=1$ such that $V,_{s s}(\sigma, s)=V,_{\sigma \sigma}(\sigma, 0)[21]$.

The Friedmann equations together with Eqs. (28) and (29) can be used to construct the potential given the background equation of state $\epsilon_{1}(\mathcal{N})$ :

$$
\begin{aligned}
V(\sigma, 0)= & -M_{\mathrm{Pl}}^{2}\left(\epsilon_{1}(\mathcal{N}-1)\right) H^{2}(\mathcal{N}) \\
= & -3 M_{\mathrm{Pl}}^{2} H_{\mathrm{end}}^{2}\left((\mathcal{N}+1)^{\alpha_{1}}-1\right) \\
& \times \exp \left(-2 \mathcal{N}+2 \int_{\mathcal{N}}^{0} \frac{d \mathcal{N}}{3(\mathcal{N}+1)^{\alpha}-1}\right),
\end{aligned}
$$

where $\mathcal{N}$ can be replaced by the background scalar field $\sigma$ using the relation

$$
\begin{aligned}
\frac{\sigma-\sigma_{\text {end }}}{M_{\mathrm{Pl}}} & = \pm \int_{\mathcal{N}}^{0} \sqrt{2 \epsilon_{1}}\left(\epsilon_{1}-1\right)^{-1} d \mathcal{N} \\
& = \pm \sqrt{6} \int_{\mathcal{N}}^{0} \frac{(\mathcal{N}+1)^{\alpha_{1} / 2}}{3(\mathcal{N}+1)^{\alpha_{1}}-1} d \mathcal{N} .
\end{aligned}
$$

For example, for $\alpha_{1}=1$ we have

$$
V(\sigma, 0) \simeq-3 M_{\mathrm{Pl}}^{2} H_{\mathrm{end}}^{2}\left(\sigma^{2} / M_{\mathrm{Pl}}^{2}-1\right) \exp \left(-2 \sigma^{2} / M_{\mathrm{Pl}}^{2}\right) .
$$

Here we set without loss of generality $\sigma_{\text {end }}=1$ and assumed $\sigma-\sigma_{\text {end }}>0$ during the smoothing phase. For all $\alpha>0$, the potential $V(\sigma, 0)$ takes the same generic form: a steep negative potential that reaches a minimum before $\sigma$ approaches $\sigma_{\text {end }}$, the standard shape potential proposed for ekpyrotic and cyclic scenarios. [This can be checked by computing the derivative of Eq. (40), $d V / d \mathcal{N}$ for different $\alpha$ and by observing from Eq. (41) that the transformation from $\mathcal{N}$ to $\sigma, \mathcal{N}(\sigma)$, is strictly monotonic.]

This means that the potential picture gives the same simple result as the model-independent hydrodynamic analysis, namely that the scale-free cyclic theory has only a single class of models all requiring a single dimensionful parameter $H_{\text {end }}^{2}$ to yield the correct spectrum of primordial density fluctuations, $\delta \rho / \rho \sim 10^{-5}$. Hence, both pictures lead to the conclusion that there is no unlikeliness problem and no extra parameters or fine-tuning problem can arise.

\section{DISCUSSION}

In this paper, our aim has been to study different cosmological scenarios in a model-independent way that does not refer directly to fields or potentials. Using a hydrodynamic approach, we derived algebraic forms for the equation-of-state parameter consistent with the scalefree principle for both inflationary and cyclic theory. In this section we discuss both theoretical and observational implications of this work.

Let us first consider inflationary cosmology alone. We found that, based on our hydrodynamical analysis, inflationary scale-free models divide into three distinct classes and identified a range of related problems: an initial conditions problem for the plateaulike and intermediate classes, and an unlikeliness problem and a fine-tuning problem for the plateau-like class. The spectrum becomes even more divided when we translate the three cases into scalar-field potentials. Hence, even limiting ourselves to scale-freeness, there is a diversity of inflationary models and predictions.

In applying the same hydrodynamic analysis to cyclic scenarios, we found cyclic theory allows only a single scale-free class of models and does not suffer from the initial conditions or unlikeliness-type problems identified for inflation. At the perturbative level, current versions of cyclic theory require a two-component fluid for the 
generation of primordial isocurvature fluctuations, which are then converted into density fluctuations. This added condition compared to inflation appears to have no disadvantage in a hydrodynamical treatment assuming scalefreeness: there were no more parameters, fine-tuning, or other kinds of constraints compared to the inflationary onefluid mechanism. Remarkably, translating this single cyclic class into scalar-field potentials, we found the same simple result.

One might ask if the problems found for inflation that were not found for cyclic may be related to the fact that a single fluid was assumed in the first case but not the second. The answer is no. As we discussed above in Sec. II, in scale-free scenarios the background is always described by a single fluid component and the presence of multiple components becomes relevant only at perturbative level. However, the inflationary problems arise at background level such that adding multiple fluid components makes (at best) no difference whatsoever. In fact, the situation for inflation is typically made worse. For example, there is a well-known two-component fluid version of inflationary theory, known as the curvaton model [35]. As in the cyclic model, the background evolution is governed by one fluid component, the inflaton, and the perturbations are controlled by another, the curvaton. Since the inflaton must satisfy the same conditions on the equation of state as in the single-fluid case, there is no change whatsoever in the problems encountered by introducing the curvaton. Since both fluids are capable of generating density perturbations, extra fine-tuning is required to regulate the interplay of the inflaton and curvaton in order that only the curvaton affects the evolution of perturbations. That is, a curvaton is not automatically the leading order contributor to the perturbations; the model must be adjusted to make it so. In particular the curvaton construction requires setting $\epsilon_{1}(N)$ for the inflaton different from $\epsilon_{2}(N)$ for the curvaton, which explicitly breaks background scale-freeness. This is qualitatively different from the cyclic case where two fluids are required to generate the leading order contribution to the density perturbations and $\epsilon_{1}(\mathcal{N})$ can be set equal to $\epsilon_{2}(\mathcal{N})$, preserving scale-freeness, as was done in Sec. IV B.

Finally, we relate our theoretical findings to current observations, in particular to recent Planck satellite measurements [2]. We see that strictly scale-free versions of both cosmological scenarios are observationally disfavored. The strictly scale-free $\phi^{4}$-chaotic inflation potential is observationally disfavored by more than $4 \sigma$ as a result of constraints on $n_{S}$ and $r$. The strictly scale-free cyclic model is consistent with current bounds on $r$ but predicts $n_{S}-1 \simeq-0.01$, which is disfavored by $3 \sigma$. That means consistency with current observational data requires some deviation from strict scale-freeness in both scenarios.

In the cyclic theory the observational value of $n_{S}-1$ can be obtained simply by introducing a very weak breaking of scale-freeness at the perturbative level $\left(\beta_{2}\right.$ slightly different from 1 or, equivalently, $|\kappa-1| \ll 1$ ) while leaving the dominant fluid and the background strictly scale-free $\left(\beta_{1}=1\right)$. In inflation, by contrast, the current observations favor scale-freeness only for plateaulike models, which suffer from the initial conditions and unlikeliness problems described above. The only power-law-like models that are not strongly disfavored require significant breaking of scale-freeness $[|\beta-1| \sim \mathcal{O}(1)]$.

What will future observations tell us about scale-free primordial cosmology? Scale-free inflation is already in serious jeopardy given what we know: there are the historic entropy $[25,36]$ and multiverse $[37,38]$ problems that apply to all inflationary models [39]. Hence, at best, we have these problems to overcome. However, future observations could make matters worse for scale-free inflation. We summarize all possible scenarios in Table I.

An important prediction for scale-free inflation that stems from this work is that the tensor-to-scalar ratio $r$ should exceed 0.0001, which is within conceivable experimental sensitivity. (Here, as throughout the paper, we assume $c_{s}>1 / 3$, as implied by current observations [3]) This bound arises because smaller $r$ requires $\alpha>3$, which, in turn, requires $n_{S}<0.95$ in disagreement with

TABLE I. Testing scale-free primordial cosmology with measurements of the tensor-to-scalar ratio $r$ and the tilt $n_{s}-1$. See discussion in text.

\begin{tabular}{|c|c|c|c|}
\hline$r$ & $n_{S}-1$ & $\begin{array}{l}\text { Unlikeliness } \\
\text { problem }\end{array}$ & Favored model \\
\hline \multirow{3}{*}{$\gtrsim 10^{-4}$} & \multirow{2}{*}{$\begin{array}{c}\text { Scale-free satisfying Eq. (25) } \\
\text { with }|\beta-1| \ll 1\end{array}$} & No, if $r \gtrsim 0.1$ & \multirow[t]{2}{*}{ Scale-free inflation } \\
\hline & & Yes, if $0.1 \gtrsim r \gtrsim 10^{-4 *}$ & \\
\hline & Violating Eq. (25) & & \\
\hline \multirow{2}{*}{$\lesssim 10^{-4}$} & \multirow{2}{*}{$\begin{array}{c}\text { Scale-free satisfying Eq. (36) } \\
\text { with }|\kappa-1| \ll 1\end{array}$} & No & Scale-free cyclic theory \\
\hline & & \multicolumn{2}{|c|}{$?$} \\
\hline
\end{tabular}

\footnotetext{
Note that the results from our model-dependent analysis in Sec. V based on scalar fields and potentials further divide plateaulike models into two groups: $\alpha \leqslant 2$, which requires $r \gtrsim 0.004$; and $2<\alpha$, which requires $10^{-4} \lesssim r \lesssim 0.004$, where this latter group requires more dimensionful parameters and has a more severe unlikeliness problem.
} 
current measurements of the spectral tilt. Note that the tensor-to-scalar ratio $r$ does not depend on the energy scale of inflation since it precisely cancels from the ratio. Models with $r$ far below $10^{-4}$ either violate existing observational constraints (such as the limit on $n_{S}-1$ ) and/ or introduce extra parameters that strongly break scalefreeness. If none of the scale-free combinations of $\left(r, n_{S}-1\right)$ is found observationally, scale-free inflation is ruled out. If one of these combinations is observed with $10^{-4}<r \lesssim 0.1$, then scale-free inflation is possible, but it is necessary to resolve the initial conditions and unlikeliness problems discussed here. If a combination is found with $r>0.1$, scale-free inflation without either of these problems is possible (though there would remain the entropy and multiverse problems common to all inflationary models).

The current situation is that observations indicate $r<0.1$. Hence, unless future $B$-mode measurements bring a surprise that overrules this result, the only possible scalefree inflationary models remaining encounter the initial conditions and unlikeliness problems discussed here.

Alternatively, future observations could find that the measured values of $r$ and $n_{S}-1$ yield no scale-free combination consistent with Eq. (25), or $r<0.0001$. Either case would eliminate all scale-free inflationary models and force extra degrees of freedom that allow virtually any outcome for $n_{S}-1$ and $r$, as exemplified by the scale-freeness violating model of Ferrara et al. [11]. In this case, inflationary cosmology loses all predictive power.

As for scale-free cyclic models, the situation is somewhat different. There is no multiverse problem and the initial conditions and unlikeliness problems found for inflation are evaded. Observationally, the strictly scale-free cyclic case $(\alpha=1)$ is disfavored because of the current constraints on the spectral tilt. A best fit to the tilt requires a small deviation from scale-freeness at the perturbative level, by setting $\beta_{2}$ (or, equivalently, $\kappa$ ) slightly greater than 1 instead of equal to 1 precisely. The forthcoming measurements of $r$ are crucial to scale-free cyclic models because all predict no observable tensor modes. Detection of primordial gravitational waves would eliminate the entire spectrum of models. On the other hand, if there is no detection and $r$ is proven to be less than 0.0001 - the conditions that eliminate scale-free inflation-scale-free cyclic would fit perfectly.

In the cyclic models considered here, we have assumed an entropic mechanism with two fluids for generating curvature perturbations. At least in currently known examples in which this is achieved with two scalar fields, the models generate non-negligible $f_{\mathrm{NL}}$ or $g_{\mathrm{NL}}$ or both. Current observational limits are consistent with predictions without requiring any additional tuning of parameters [30], but future measurements could result in detection or tighter constraints. Although non-Gaussianity is not directly predicted by hydrodynamical analysis and is more model dependent in cyclic models, future measurements could be useful in distinguishing inflation versus cyclic scenarios and the testing the hypothesis of scale-free primordial cosmology.

In sum, introducing the scale-free principle makes cosmological theories-both inflationary and cyclicmeaningfully predictive and allows for observational test. Both for scale-free inflationary and cyclic cosmology, we could identify all combinations of parameters $\left(r, n_{S}-1\right)$ consistent with the theory. If such a combination is not measured, the theory is falsified. Most interestingly, forthcoming measurements are capable of testing and eliminating scale-free inflationary models, scale-free cyclic models, or both, as indicated by the "?" in Table I. Eliminating both means relinquishing scale-freeness and having to settle for unpredictive theory, like [11], or seeking another type of cosmological theory that retains scale-freeness and predictive power.

\section{ACKNOWLEDGMENTS}

This work was inspired in part by a talk and recent paper by V. Mukhanov [13]. We thank J. Khoury, J.-L. Lehners, and $\mathrm{N}$. Turok for very helpful comments on the manuscript. This research was partially supported by the U.S. Department of Energy under Grant No. DE-FG0291ER40671 (P. J. S.), by NSF Grant No. AST-0907890 and NASA Grants No. NNX08AL43G and No. NNA09DBB30A (A. L.), and the Perimeter Institute for Theoretical Physics (A. I. and P. J. S.). Research at Perimeter Institute is supported by the Government of Canada through Industry Canada and by the Province of Ontario through the Ministry of Research and Innovation. A. I. also acknowledges the support of the European Research Council via the Starting Grant No. 256994. The work of A.I. is supported in part by a grant from the John Templeton Foundation. A. I. thanks the Physics Department of Princeton University for hospitality while this research was completed.

\section{APPENDIX: DERIVATION OF EQ. (31)}

In order to derive the general hydrodynamic expression for the spectral tilt of primordial density fluctuations in cyclic theories, we follow the same procedure as for inflation [23]. Namely, we first solve for the perturbations, assuming the fluids can be represented as scalar fields with potentials, and then we convert the potential parameters in the expression derived for the tilt into hydrodynamic variables. To represent the two-component fluid we choose two fields, $\sigma$ and $s$, where $\sigma$ corresponds to the fluid component governing the background evolution described by equation of state $\epsilon_{1}$ and $s$ is the field representing the fluid that generates the isocurvature fluctuations before the bounce that are later converted to curvature perturbations during the bounce. The second fluid has equation-of-state parameter $\epsilon_{2}$. The perturbation equation is given by 


$$
\delta \ddot{s}+3 \mathrm{H} \delta \dot{s}+\left(\frac{k^{2}}{a^{2}}+V,_{s s}\right) \delta s=0,
$$

where a dot denotes derivation with respect to physical time and $k$ is the adiabatic mode.

For the cyclic potential we choose the form

$$
V(\sigma, s)=V(\sigma, 0)\left(1+\frac{1}{2} \kappa \frac{V, \sigma \sigma}{V(\sigma, 0)} s^{2}+\mathcal{O}\left(s^{3}\right)\right),
$$

in agreement with [21,30]. Here $\kappa$ is the ratio of the equation-of-state parameters, $\kappa \equiv \epsilon_{2} / \epsilon_{1}$ as in Eq. (32). $V(\sigma, s)$ is constructed such that for $\kappa=1$ it yields scale-free solutions; this corresponds to the case $V(\sigma, s){ }_{s s}=V(\sigma, 0){ }_{\sigma \sigma}$. Parameterizing the cyclic potential in this way is useful since the form naturally incorporates the entropic mechanism by dividing the potential into a first factor, that describes the background evolution along the $\sigma$ direction, and the second factor, which describes the direction of the isocurvature perturbations. Furthermore, this form encompasses all known simple cyclic potentials, such as models that can be written as sums of exponentials of independent fields.

After rescaling $\delta S \equiv a(\eta) \delta s$ and assuming standard Bunch-Davies initial conditions, $\delta s \rightarrow e^{-i k \eta} /(2 k)^{3 / 2}$, the solution of Eq. (A1) is the Hankel function

$$
\delta s=\frac{\sqrt{-\pi \eta}}{2} H_{\nu}^{(1)}(-k \eta)
$$

with

$$
\nu^{2}=\frac{1}{4}+\eta^{2}\left(\frac{a^{\prime \prime}}{a}-a^{2} \kappa V,_{\sigma \sigma}\right) .
$$

[1] P. Ade et al. (Planck Collaboration), arXiv:1303.5076.

[2] P. Ade et al. (Planck Collaboration), arXiv:1303.5082.

[3] P. Ade et al. (Planck Collaboration), arXiv:1303.5084.

[4] J. L. Sievers et al., J. Cosmol. Astropart. Phys. 10 (2013) 060.

[5] E. R. Harrison, Phys. Rev. D 1, 2726 (1970).

[6] R. Sunyaev and Y. Zeldovich, Astrophys. Space Sci. 7, 3 (1970).

[7] P. Peebles and J. Yu, Astrophys. J. 162, 815 (1970).

[8] A. H. Guth, Phys. Rev. D 23, 347 (1981).

[9] A. D. Linde, Phys. Lett. 108B, 389 (1982).

[10] A. Albrecht and P. J. Steinhardt, Phys. Rev. Lett. 48, 1220 (1982).

[11] S. Ferrara, R. Kallosh, A. Linde, and M. Porrati, Phys. Rev. D 88, 085038 (2013).
Here a prime denotes derivative with respect to conformal time $\eta$. On large scales, $k \ll a H, \delta s \sim k^{-\nu}$. This corresponds to a spectral tilt

$$
n_{S}-1=3-2 \nu \text {. }
$$

To express the tilt in hydrodynamical language, we follow [15] and rewrite $H, a$, and $V,{ }_{\sigma \sigma}$ in terms of the background equation-of-state parameter $\epsilon_{1}(\mathcal{N})$ :

$$
\begin{gathered}
(a H)^{-1} \simeq \epsilon_{1} \eta\left(1-\frac{1}{\epsilon_{1}}-\frac{\epsilon_{1}, \mathcal{N}}{\epsilon_{1}}\right), \\
\frac{a^{\prime \prime}}{a} \simeq 2 a^{2} H^{2}\left(1-\frac{1}{2 \epsilon_{1}}\right), \\
V,_{\sigma \sigma} \simeq-H^{2}\left(2 \epsilon_{1}^{2}-6 \epsilon_{1}-\frac{5}{2}\left(\epsilon_{1}-1\right) \epsilon_{1}, \mathcal{N}\right) .
\end{gathered}
$$

After some algebra, we find

$$
\nu^{2} \simeq \frac{1}{4}+2\left(\kappa+3 \cdot \frac{1-2 \kappa}{2 \varepsilon}+\frac{8-5 \kappa}{4} \cdot \frac{\epsilon, \mathcal{N}}{\epsilon}\right)
$$

where we neglected terms of order $1 / \epsilon^{2}$. Finally, substituting into Eq. (A5) yields the hydrodynamic expression for the spectral tilt as stated in Eq. (31).
[12] J. Khoury, P. J. Steinhardt, and N. Turok, Phys. Rev. Lett. 91, 161301 (2003).

[13] V. Mukhanov, Eur. Phys. J. C 73, 2486 (2013).

[14] A. Ijjas, P. J. Steinhardt, and A. Loeb, Phys. Lett. B 723, 261 (2013).

[15] J.-L. Lehners, P. McFadden, N. Turok, and P. J. Steinhardt, Phys. Rev. D 76, 103501 (2007).

[16] J. Khoury, B. A. Ovrut, P. J. Steinhardt, and N. Turok, Phys. Rev. D 64, 123522 (2001).

[17] J. Khoury, B. A. Ovrut, N. Seiberg, P. J. Steinhardt, and N. Turok, Phys. Rev. D 65, 086007 (2002).

[18] J. M. Bardeen, P. J. Steinhardt, and M. S. Turner, Phys. Rev. D 28, 679 (1983).

[19] V. F. Mukhanov, H. Feldman, and R. H. Brandenberger, Phys. Rep. 215, 203 (1992). 
[20] P. Creminelli, A. Nicolis, and M. Zaldarriaga, Phys. Rev. D 71, 063505 (2005).

[21] E. I. Buchbinder, J. Khoury, and B. A. Ovrut, J. High Energy Phys. 11 (2007) 076.

[22] J. D. Barrow and A. R. Liddle, Phys. Rev. D 47, R5219 (1993).

[23] L.-M. Wang, V. F. Mukhanov, and P. J. Steinhardt, Phys. Lett. B 414, 18 (1997).

[24] R. H. Brandenberger, H. Feldman, and J. Kung, Phys. Scr. T36, 64 (1991).

[25] G. Gibbons and N. Turok, Phys. Rev. D 77, 063516 (2008).

[26] A. R. Liddle and D. Lyth Cosmological inflation and largescale structure, (Cambridge University Press, Cambridge, England, 2000).

[27] J. K. Erickson, S. Gratton, P. J. Steinhardt, and N. Turok, Phys. Rev. D 75, 123507 (2007).

[28] P. J. Steinhardt and N. Turok, Science 296, 1436 (2002).
[29] P. J. Steinhardt and N. Turok, Phys. Rev. D 65, 126003 (2002).

[30] J.-L. Lehners and P. J. Steinhardt, Phys. Rev. D 87, 123533 (2013).

[31] F. Bezrukov and M. Shaposhnikov, Phys. Lett. B 659, 703 (2008).

[32] C. Destri, H. J. de Vega, and N. Sanchez, Phys. Rev. D 77, 043509 (2008).

[33] K. Nakayama, F. Takahashi, and T. T. Yanagida, Phys. Lett. B 725, 111 (2013).

[34] A. Vilenkin, Phys. Rev. D 30, 509 (1984).

[35] D. H. Lyth and D. Wands, Phys. Lett. B 524, 5 (2002).

[36] R. Penrose, Ann. N.Y. Acad. Sci. 571, 249 (1989).

[37] P. J. Steinhardt, in The Very Early Universe, edited by G. Gibbons, S. Hawking, and S. Siklos (Cambridge University Press, Cambridge, England, 1983), pp. 251-266.

[38] A. Vilenkin, Phys. Rev. D 27, 2848 (1983).

[39] P. J. Steinhardt, Sci. Am. 304N4, 18 (2011). 